\title{
Biomarkers for Impending Risk of Osteoporosis in Premenopausal Women
}

\author{
Rehana Rahman ${ }^{1}$, Ali Usman², Aisha Sheikh ${ }^{3}$ and Rozeena Baig ${ }^{1}$ \\ ${ }^{1}$ Department of Biological \& Biomedical Sciences, The Aga Khan University, Karachi, Pakistan \\ ${ }^{2}$ Department of Internal Medicine, The Aga Khan University, Karachi, Pakistan \\ ${ }^{3}$ Department of Medicine, The Aga Khan University, Karachi, Pakistan
}

\begin{abstract}
Objective: To compare 'cytokines' and 'bone turnover markers' in pre- and post-menopausal women and identify their relationship with bone mineral density (BMD) in both groups.

Study Design: A cross-sectional study.

Place and Duration of Study: Department of Biological \& Biomedical Sciences, The Aga Khan University, Karachi, Pakistan, from June 2017 to August 2019.

Methodology: Groups comprised of healthy premenopausal and postmenopausal women from the general population belonging to different ethnic groups and socio-economic status. Serum cytokines and bone turnover markers were assessed by solid-phase immunoassays, BMD $\left(\mathrm{gm} / \mathrm{cm}^{2}\right)$ ] measurement was performed by dual-energy X-ray absorptiometry at the hip, lumbar spine, and proximal femur. Results were interpreted as a sum of T scores calculated by BMD of the above-mentioned sites.

Results: Cytokines and bone turnover markers were significantly high in post-menopausal women $(p<0.001)$. A negative correlation ( $r=0.32$ ) of TNF $\alpha$ with BMD (total T scores) observed in premenopausal women was found to be significant, however, no significant association of BMD was detected in post-menopausal women.

Conclusion: There is an increase in the production of cytokines and bone turnover markers after menopause. TNF- $\alpha$ follows this usual pattern of increase in post-menopausal women and can predict impending bone loss and osteopenia in premenopausal females. Therefore, estimation of this cytokine in pre-menopausal women can give a fair indication of a decline in BMD, bone health, and risk of future osteoporosis.
\end{abstract}

Key Words: Bone mineral density, Cytokines, Bone turnover markers, Osteoporosis.

How to cite this article: Rahman R, Usman A, Sheikh A, Baig R. Biomarkers for Impending Risk of Osteoporosis in Premenopausal Women. J Coll Physicians Surg Pak 2021; 31(08):910-915.

\section{INTRODUCTION}

Osteoporosis is the most common silent disease with the thirdhighest incidence in the aging population. ${ }^{1}$ The important public health problem has added an increased incidence of fractures, disabilities, deprived quality of life with increased mortality in the aging population of developing countries. ${ }^{1-3}$

Bone turnover is the process of bone resorption followed by replacement by new bone formation. Osteoclast and osteoblast are the main cells involved in this process. Osteoclasts cause bone resorption; whereas, osteoblasts take part in the formation of new bone. ${ }^{4}$

Correspondence to: Dr. Rehana Rehman, Department of Biological \& Biomedical Sciences, The Aga Khan University, Karachi, Pakistan

E-mail: rehana.rehman@aku.edu

Received: March 01, 2021; Revised: June 11, 2021;

Accepted: July 16, 2021

DOI: https://doi.org/10.29271/jcpsp.2021.08.910
One of the mechanisms responsible for communication is through cytokines. Cytokines are small proteins that facilitate interactions between cells; cytokines like interleukin-1, M-CSF, tumor necrosis factor; and interleukin- 6 can incite the formation as well as bone-resorbing capability of osteoclasts. Meanwhile, cytokines such as interleukin-4, gamma-interferon, and transforming factor-beta inhibit both osteoclast formation and osteoclast activity.Therefore, specific cytokine levels in the bone marrow microenvironment may play a crucial role in the regulation of osteoclast activity and knowledge of these cytokines can provide insight into normal calcium homeostasis and disorders of bone turnover, such as osteoporosis. ${ }^{5}$ Clinically, osteoporosis is diagnosed by using dual energy $x$-ray absorptiometry (DXA) in post-menopausal women. BMD; T-score of -2.5 or less at any observed site or history of a former fragility fracture is considered for osteoporosis. ${ }^{6}$ The loss of bone, which occurs as a result of an imbalance between the bone foundation and resorption, can be measured by BMD as well as by changes in biochemical markers like osteocalcin, C-terminal telopeptideforidentification of osteoporotic fractures. ${ }^{7}$ DXA scan however has its limitations as a costly investigation which is notavailable in all hospitals. 
Estrogen regulatesthe productionandactivity of pro-inflammatory cytokines like 'interleukin (1L)-1', 'IL-6' and 'tumor necrosis factor-alpha (TNF- $\alpha$ ). Its deficiency in postmenopausal women leads to the increased production of these cytokines which activate the genesis of osteoclasts, leading to bone loss. ${ }^{8} \mathrm{TNF}-\alpha$ along with other pro-inflammatory cytokines takes part in bone remodelling, maintenance of bone homeostasis by initiating osteoclastogenesis and restraining delineation of osteoblast. ${ }^{9}$ Osteoclast formation is regulated by TNF $\alpha$ through 'receptor activator of nuclear factor-kappa-B ligand (RANKL)', which is well known for its role in normal bone modeling and remodeling. IL- 6 expedites the activity of osteoblasts as well as osteoclasts. Both TNF- $\alpha$ and IL- 6 are responsible for immune-mediated bone diseases of which postmenopausal osteoporosis and rheumatoid arthritis are worth mentioning. ${ }^{10}$

Markers of bone metabolism are innovative tools that perceive the process of bone remodelling concerning its configuration and resorption. The wider accessibility of dependable, economical, and specific assays for bone turnover would supplement the BMD measurement in the detection, management, and follow-up of osteoporosis. Therefore, keeping in mind, the growing prevalence of osteoporosis and its impact on the health and economy of Pakistan. It was therefore, hypothesised that loss of minerals in bones can be detected by increased levels of proinflammatory cytokines and bone turnover markers.

The objective of this study was to explore the association of cytokines and bone turnover markers with the probable risk of osteoporosis by comparison of these markers in pre-and post-menopausal women and identify the relationship of these biomarkers with bone mineral density (BMD).

\section{METHODOLOGY}

This cross-sectional study was conducted from June 2017 to August 2019 at the Department of Biological and Biomedical Sciences, The Aga Khan University, Karachi, Pakistan, according to Helsinki Declaration next to endorsement by 'Ethical Review Committee of The Aga Khan University 4146BBS-ERC-16'. Sample size calculation was calculated by 'Open Epi sample size calculator' with a $99 \%$ confidence interval and $5 \%$ margin of error.

$$
n=\frac{2 \sigma^{2}\left(Z_{\beta}+Z_{\alpha 2}\right)^{2}}{\text { difference }^{2}}
$$

The sample size was estimated to be $162 .{ }^{11}$ Pre- and post-menopausal women with the age ranging from 20 to 70 years were selected from the outdoor patient clinic of The Aga Khan University and the community. Healthy female subjects; post-menopausal women; 5 years of menopause or greater and premenopausal women with regular cycles (20-45 years of age) of all ethnic groups and socioeconomic status were included. Women with 'metabolic diseases, hyperthyroidism, hyperparathyroidism, Diabetes mellitus, autoimmune diseases, history of premature menopause ( $<40$ years), cirrhosis, hepatitis and hepatoma' were excluded from the study. Women with a history of intake of drugs like bisphosphonates, calcitonin, anticonvulsants, glucocorticoids, vitamin D, calcium and hormonal replacement therapy, history of a previous fracture, and smokers were also excluded.

After taking informed consent, history, and physical examination, blood samples were collected. Bone density measured as $\mathrm{g} / \mathrm{cm}_{-}^{2}$ was converted into reference values for age as T scores.

\section{$T$ score $=\frac{\text { patient's } B M D \text {-poptlation peak BMD }}{\text { standard deviation }(S D) \text { of popslation peak BMD }}$}

Details of Kits: Serum interleukin 6 was determined by using a commercially available Kit Cat \# BMS213-2by Bioassay Technology Laboratory. The sensitivity of the assay was $0.03 \mathrm{pg} / \mathrm{m}$ L. Serum Tumor necrosis factor Alpha (TNF- $\alpha$ ) was analyzed by "Enzyme immunoassay Kit Cat No E $0082 \mathrm{Hu}$ " by Bioassay Technology Laboratory. The sensitivity of the assay was $1.52 \mathrm{ng} / \mathrm{L}$. Osteocalcin was measured by "EIA kit Catalogue no EI555Hu by Bioassay Technology Laboratory. Assay sensitivity was 0.026 $\mathrm{ng} / \mathrm{ml}$. serum human cross-linked carboxy-terminal telopeptides type I collagen was done by using Kit-Cat No. E6465Hu (Bioassay Technology Laboratory). The sensitivity of the assay was $0.17 \mathrm{ng} / \mathrm{ml}$. The biomarkers (serum cytokines, osteocalcin, carboxytelopeptide, serum interleukin 6 (IL-6), serum TNF- $\alpha$ was performed as permanufacturers protocol

Data was initially entered in Excel, imported to statistical software SPSS version 19.0. Quantitative parameters were expressed as mean \pm S.D. The quantitative variables were compared based on the Student t-test. Pearson correlation coefficient was used ( $p$-value of $<0.05$; statistically significant) to determine the association of differentstudy parameters.

\section{RESULTS}

Table I compares demographic, bone turnover markers, and cytokines in pre-menopausal $(n=60)$ and post-menopausal (102) female subjects. TNF $\alpha$ and IL- 6 was higher in post-menopausal females $81.52 \pm 13.87 v s$. $7.26 \pm 1.36 ; p<0.001$ ) and $10.64 \pm 3.12$ vs. $1.33 \pm 0.24 ; p<0.001$ ). The bone turnover markers Osteocalcin and Carboxytelopeptide were significantly higher in the post-menopausal group $(p<0.001)$.

Table II represents the correlation of DXA scores in premenopausal and post-menopausal groups of subjects with study variables. A significant negative correlation $(r=0.32)$ of BMD was observed with TNF $\alpha$ in premenopausal women. When compared with bone density measured at various sites (Table III), it was observed that TNF- $\alpha$ correlated with BMD at the hip ( $r=0.372$, $p<0.001)$ and femoral neck $(r=-0.269,<0.05)$. Figure $1 a$ and $1 b$ represent the correlation of TNF $\alpha$ with BMD scores in premenopausal and postmenopausal females. The correlation of age with BMD in both groups is represented in Figure $2 a$ and $2 b$. 
Table I: Biophysical and biochemical variables in premenopausal and postmenopausal women.

\begin{tabular}{|c|c|c|c|}
\hline & Premenopausal & Postmenopausal & \multirow{3}{*}{ p-value } \\
\hline & women $(n=60)$ & women $(n=102)$ & \\
\hline & Mean \pm S. D & Mean \pm S.D & \\
\hline Age (years) & $32.63 \pm 7.263$ & $53.22 \pm 5.616$ & $p<0.001$ \\
\hline $\mathrm{BMI} \mathrm{kg} / \mathrm{m}^{2}$ & $23.2 \pm 4.51$ & $28.37 \pm 4.91$ & $p<0.001$ \\
\hline T scores & $-1.37 \pm 0.95$ & $-1.47 \pm 0.97$ & 0.524 \\
\hline \multicolumn{4}{|l|}{ Bone formation marker } \\
\hline Serum osteocalcin & $10.21 \pm 5.88$ & $20.79 \pm 11.73$ & $p<0.001$ \\
\hline Serum carboxytelopeptide (CTX) & $6.26 \pm 4.47$ & $30.71 \pm 18.97$ & $<0.001$ \\
\hline \multicolumn{4}{|l|}{ Serum Cytokines } \\
\hline Tumor necrosis factor alpha [(TNF- $\alpha)(\mathrm{pg} / \mathrm{ml})]$ & $7.26 \pm 1.37$ & $81.52 \pm 13.87$ & $<0.001$ \\
\hline Interleukin $6[(\mathrm{IL}-6)(\mathrm{pg} / \mathrm{ml})]$ & $1.33 \pm 0.24$ & $10.64 \pm 3.12$ & $<0.001$ \\
\hline
\end{tabular}

Table II: Correlation of T scores with proinflammatory cytokines and bone turnover markers in pre- and post-menopausal females.

\begin{tabular}{|c|c|c|}
\hline Study variables & Premenopausal females & Postmenopausal females \\
\hline Age (years) & $-.364(* *)$ & $-.545(* *)$ \\
\hline $\mathrm{BMI} \mathrm{kg} / \mathrm{m}^{2}$ & 0.163 & 0.139 \\
\hline Tumor necrosis factor alpha (TNF- $\alpha$ ) & $-.324(*)$ & 0.006 \\
\hline Interleukin 6(IL-6) & 0.069 & -0.06 \\
\hline Osteocalcin & 0.15 & 0.113 \\
\hline Carboxytelopeptide (CTX) & -0.092 & -0.03 \\
\hline
\end{tabular}

Table III: Correlation of T scores at 3 sites with proinflammatory cytokines and bone turn over markers in pre and post-menopausal females.

\begin{tabular}{|l|l|l|l|l|l|l|}
\hline \multirow{2}{*}{ Variables } & Hip & Spine & Femoral Neck & Post & Pre \\
\cline { 2 - 7 } & Pre & Post & Pre & Post & $-.269(*)$ \\
\hline Tumor necrosis factor alpha (TNF- $\alpha$ ) & $-.372(* *)$ & 0.005 & -0.241 & -0.011 & 0.027 \\
\hline Interleukin 6 (IL-6) & 0.064 & -0.064 & 0.042 & -0.025 & 0.084 \\
\hline Osteocalcin & 0.128 & 0.055 & 0.156 & 0.094 & 0.131 \\
\hline Carboxytelopeptide (CTX) & -0.062 & -0.034 & -0.131 & -0.079 & -0.061 \\
\hline Correlation is significant at 0.05 (2-tailed). Correlation is significant at the.001 level (2-tailed). & 0.153 & 0.052 \\
\hline
\end{tabular}

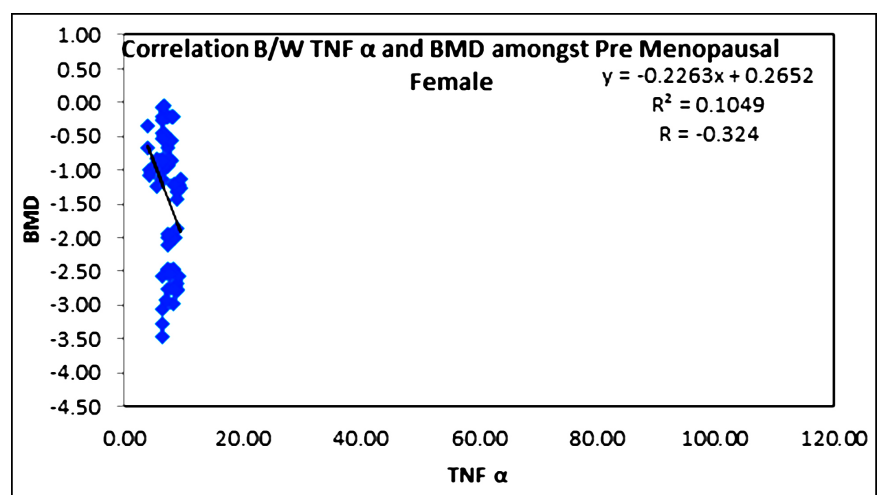

Figure 1a: Correlation of bone mineral density scores with TNF- $\alpha$ in pre-menopausal females.

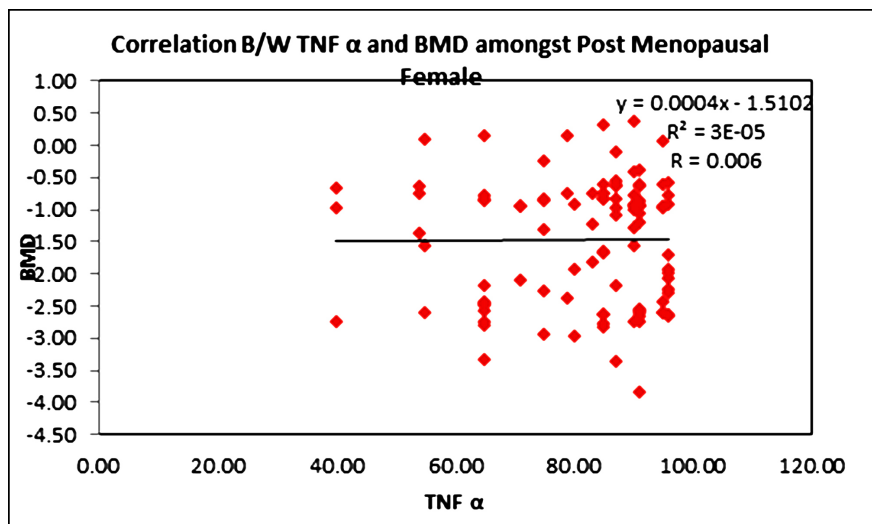

Figure 1b: Correlation of bone mineral density scores with TNF- $\alpha$ in post-menopausal females.

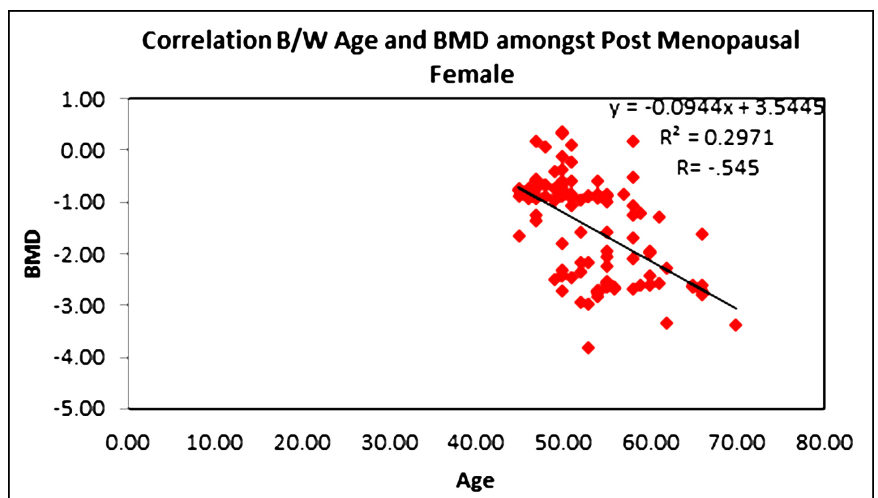

Figure 2a: Correlation of bone mineral density scores with age in pre-menopausal females.

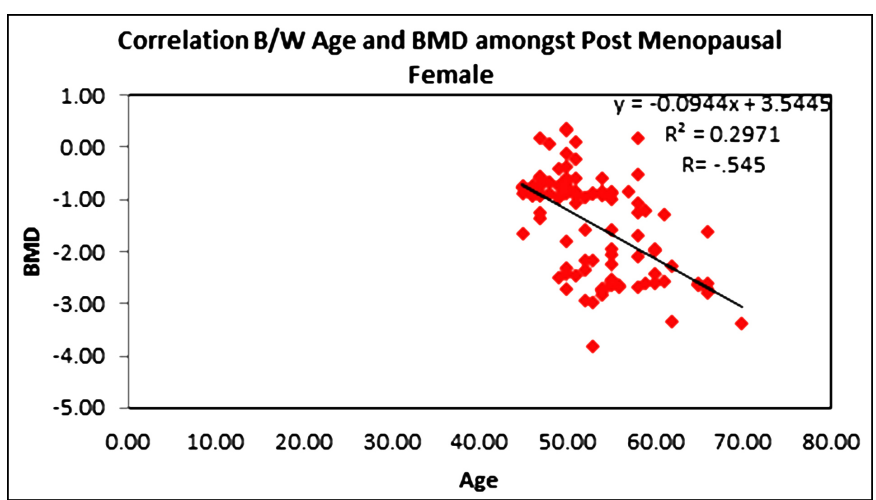

Figure 2b: Correlation of bone mineral density scores with age in postmenopausal females. 


\section{DISCUSSION}

Menopause is a phase of changeover in the female reproductive cycle that has an impact on bone health and mineral metabolism. As ovarian function declines with menopause, there is a rise of pro-inflammatory cytokines leading to post-menopausal bone loss and osteoporosis. Postmenopausal osteoporosis is a point of concern for clinicians as well as epidemiologists due to its increased incidence and the forthcoming burden on the health economy in low-middle-income countries. The new emerging clinical and molecular evidence suggests a link of inflammation in the etiopathogenetic mechanisms leading to osteoporotic fractures. The role of pro-inflammatory cytokines in the acceleration of bone loss has thus been documented in postmenopausal women. ${ }^{12}$ In addition to that, the hormonal imbalance exerts physiological and biochemical changes, which increase the bone turnover in these females which was manifested by reduced BMD in our research results.

Specific cytokine levels in the bone homeostasis play a crucial role in the regulation of osteoclast activity and therefore comprehension of these cytokines can provide insight into normal calcium homeostasis and conditions of bone turnover like osteoporosis. ${ }^{5}$ It was observed high TNF- $\alpha$ levels in post-menopausal females which supports the fact that TNF- $\alpha$ is an imperative mediator of bone resorption ${ }^{13}$ which increases osteoclast differentiation, inhibits osteoblast survival and therefore leading to low BMD. ${ }^{14} A$ three years study on the effect of TNF- $\alpha$ blocking therapy on bone formation and bone resorption documented an increase in bone formation marker; bone-specific alkaline phosphatase (BALP) which causes bone mineralization process and collagen synthesis. ${ }^{15}$ In addition that bone resorption markers CTX was reduced due to the blocking effect of TNF- $\alpha$ caused due to a reduction in collagen degradation. ${ }^{15}$ The role of Inflammation in the revelation of osteoporosis by increased interleukin levels in post-menopausal women was observed by an increase in IL-6 in this study, which is comparable to a study done by Jeedigunta et al. ${ }^{8}$ However, no relationship between bone-resorbing cytokines and ovarian function was observed by other researchers.

The high TNF- $\alpha$ and IL- 6 observed in post-menopausal females regarding the role of IL- 6 and TNF- $\alpha$ in bone resorption and formation processes are documented. In a study, women in both pre- and post-menopausal groups were osteopenic $^{16}$ and a significant negative co-relation of TNF- $\alpha$ with BMD was noticed in premenopausal women. It is expected estrogen deficiency in postmenopausal women and loss of ovarian function indorses signaling and gene expression cascade of series of pro-inflammatory cytokines which stimulates macrophage colony-stimulating factor (MCSF) as well as IL-6. ${ }^{17}$ Recent literature suggests that exogenous TNF- $\alpha$ aggravates endoplasmic reticulum stress, decreases collagen secretion, and prompts apoptosis and inflammatory response in human osteoblast-like SaOs-2 cells. Improvement in bone formation and lumbar spine BMD was observed with anti-TNF- $\alpha$ therapy. ${ }^{15}$ The finding highlights the consideration of the association of TNF $\alpha$ with osteopenia for risk assessment of future osteoporosis and bone health.

Aging is also a contributing factor to increased levels of proinflammatory cytokines and low BMD. ${ }^{18,19}$ Hence, the authors observed low BMD with advancing age. This observation is supported by other studies done in elderly postmenopausal women. ${ }^{18,20}$ Studies have suggested low BMI responsible for osteoporosis and accompanying fractures. ${ }^{21,22}$ This can be explained by the fact that a larger body mass enforces mechanical load on the bone which increases the bone mass as compensation. A study was done on Qatari women nevertheless revealed greater BMD in an obese group with its positive association with BMD at spinal and femoral sites. ${ }^{23}$ It was observed high BMI in post-menopausal females without any significant association with BMD supported by literature. ${ }^{24}$

An increase in bone turnover irrespective of the cause leads to weakening of bone microarchitecture; hence, contributing to an increased risk of fracture which is independent of low BMD. Osteocalcin and CTX levels were high in post-menopausal women in this study, which is supported by another study. ${ }^{17}$ This study highlights the role of cytokines especially TNF- $\alpha$ in the perimenopausal stage as the probable link of inflammation leading to osteopenia. An inclusive evaluation of inflammatory biomarkers and related metabolite pathways can help in determining bone outcomes, predict subacute stage after fractures, which can be helpful to reduce bone loss, maintain bone density, and diminish susceptibility of secondary fractures.

TNF- $\alpha$ though detected as a biomarker for osteopenia in premenopausal women; its utility in predicting osteoporosis needs more research evidence and inclusion of osteoporotic females with high $T$ scores. The authors have also not taken the effect of other hormones which impact calcium homeostasis and bone turnover in our study. However, the strength of the study is a suggestion for consideration of high TNF- $\alpha$ (detected due to any other cause) for evaluation of BMD, irrespective of the reproductive age of a woman.

\section{CONCLUSION}

There is an increase in the production of cytokines and bone turnover markers after menopause. TNF- $\alpha$ though follows this usual pattern of increase in post-menopausal women, yet can predict impending bone loss and osteopenia in premenopausal females. Therefore, estimation of this cytokine in pre-menopausal women can give a fair indication of a decline in BMD, bone health, risk of future osteoporosis, and plan of preventive measures to be taken before the onset of menopause.

\section{RECOMMENDATION:}

The authors recommend BMD be performed in case an 
increase in TNF- $\alpha$ is detected due to other causes in male and female subjects to identify the risk of osteoporosis.

\section{ACHNOWLEDGMENT:}

The authors are thankful to the HEC- NRPU Grant Section and the Department of Biological \& Biomedical Sciences for the facilitation of the project. Authors' special thanks to Dr. Najeeha Talat Iqbal for her support in the write-up of the grant proposal, Dr. Rafat Murad for collaboration and recruitment of subjects, and Miss Mussarat Ashraf for support in bench work undertaken in the Department of Biological \& Biomedical Sciences.

\section{FUNDING:}

HEC--NRPU project\# 5215/Sindh/NRPU/R\&D/HEC/2016.

\section{ETHICAL APPROVAL:}

Ethical approvals were obtained prior to initiation of the research work from Ethical Review Committee of The Aga Khan University; 4146- BBS-ERC-16.

\section{PATIENTS' CONSENT:}

Informed consents were obtained from the patients to conduct the study and publish the data of the research.

\section{CONFLICT OF INTEREST:}

The authors declared no conflict of interest.

\section{AUTHOR'S CONTRIBUTION:}

RR, AS: Designed the study and applied for HEC grant. $A U, R B$ : Helped in recruitment of subjects.

All authors took part in acquisition, analysis and interpretation of data. They took part in preparation of manuscript, critically analysed the content and approved the manuscript before submission.

\section{REFERENCES}

1. Aziziyeh R, Garcia Perlaza J, Saleem N, Sadat-Ali M, Elsalmawy A, McTavish RK, et al. The burden of osteoporosis in Saudi Arabia: A scorecard and economic model. J Med Econ 2020; 23(7):767-75. doi: 10.1080/13696998.2020.1737536.

2. Bassatne A, Harb H, Jaafar B, Romanos J, Ammar W, El-Hajj Fuleihan G. Disease burden of osteoporosis and other non-communicable diseases in Lebanon. Osteoporos Int 2020; 31(9):1769-77. doi: 10.1007/s00198-020-05433-w.

3. Marcellusi A, Rotundo MA, Nardone C, Sciattella P, Gazzillo $S$, Rossini $M$, et al. Osteoporosis: economic burden of disease in Italy. Clin Drug Investig 2020; 40(5):449-58. doi: 10.1007/s40261-020-00904-8.

4. Burch J, Rice S, Yang H, Neilson A, Stirk L, Francis R, et al. Systematic review of the use of bone turnover markers for monitoring the response to osteoporosis treatment: the secondary prevention of fractures, and primary prevention of fractures in high-risk groups. Health Technol Assess 2014; 18(11):1-180. doi: 10.3310/hta18110.

5. Chen X, Wang Z, Duan N, Zhu G, Schwarz EM, Xie C. Osteoblast-osteoclast interactions. Connect Tissue Res 2018; 59(2):99-107. doi: 10.1080/03008207.2017.1290085.
6. Kanis JA. Assessment of fracture risk and its application to screening for postmenopausal osteoporosis: synopsis of a WHO report. Osteoporos Int 1994; 4(6):368-81. doi: 10.1007/BF01622200.

7. Mithal A, Bansal B, Kyer CS, Ebeling P. The Asia-pacific regional audit-epidemiology, costs, and burden of osteoporosis in India 2013: a report of international osteoporosis foundation. Indian journal of endocrinology and metabolism. Indian J Endocrinol Metab 2014; 18(4):449-54. doi: 10.4103/2230-8210.137485.

8. Jeedigunta $Y$, Sultana S, Kalashikam RR, Manchala R. Systemic Inflammation: A Risk Factor for Osteoporosis in Pre-and Post-menopausal Women. J Women's Health Dev 2020;3(4):423-31. DOI: 10.26502/fjwhd.2644-28840050.

9. Osta B, Benedetti G, Miossec P. Classical and paradoxical effects of TNF- $\alpha$ on bone homeostasis. Front Immunol 2014; 5:48. doi: 10.3389/fimmu.2014.00048. eCollection 2014.

10. Wang T, He C. TNF- $\alpha$ and IL-6: The Link between Immune and Bone System. Curr Drug Targets 2020; 21(3):213-227. doi: $10.2174 / 1389450120666190821161259$.

11. Rosen CJ, Chesnut III CH, Mallinak NJ. The predictive value of biochemical markers of bone turnover for bone mineral density in early postmenopausal women treated with hormone replacement or calcium supplementation. Clin Endocrinol Metab 1997; 82(6):1904-10. doi: 10.1210/ jcem.82.6.4004.

12. Al-Daghri NM, Yakout S, Al-Shehri E, Al-Fawaz H, Aljohani N, Al-Saleh Y. Inflammatory and bone turnover markers in relation to PTH and vitamin D status among Saudi postmenopausal women with and without osteoporosis. Int J Clin Exp Med 2014; 7(9):2812-9. eCollection 2014.

13. McLean RR. Proinflammatory cytokines and osteoporosis. Curr Osteoporos Rep 2009; 7(4):134-9. doi: 10.1007/ s11914-009-0023-2.

14. Lee SH, Kim TS, Choi Y, Lorenzo J. Osteoimmunology: cytokines and the skeletal system. BMB Rep 2008; 41(7):495-510. doi: 10.5483/bmbrep.2008.41.7.495.

15. Arends S, Spoorenberg A, Houtman PM, Leijsma MK, Bos R, Kallenberg CG, et al. The effect of three years of TNF alpha blocking therapy on markers of bone turnover and their predictive value for treatment discontinuation in patients with ankylosing spondylitis: a prospective longitudinal observational cohort study. Arthritis Res Ther 2012; 14(2):R98. doi: 10.1186/ar3823.

16. Ho-Pham LT, Nguyen UD, Pham HN, Nguyen ND, Nguyen TV. Reference ranges for bone mineral density and prevalence of osteoporosis in Vietnamese men and women. BMC MusCuloskelet Disord 2011; 12:182. doi: 10.1186/14712474-12-182.

17. Al-Daghri NM, Aziz I, Yakout S, Aljohani NJ, Al-Saleh Y, Amer $\mathrm{OE}$, et al. Inflammation as a contributing factor among postmenopausal Saudi women with osteoporosis. Medicine (Baltimore) 2017; 96(4):e5780. doi: 10.1097/MD.0000000000http://pubmed.ncbi.nlm.nih.gov/28121926/\#005780.

18. Murad R, Shezad Z, Ahmed S, Ashraf M, Qadir M, Rehman R. Serum tumour necrosis factor alpha in osteopenic and osteoporotic postmenopausal females: A cross-sectional study in Pakistan. JPMA J Pak Med Assoc 2018; 68(3):428-31.

19. JafariNasabian P, Inglis JE, Kelly OJ, llich JZ. Osteosarcopenic 
obesity in women: impact, prevalence, and management challenges. Int J Womens Health 2017; 9:33-42. doi: 10.2147/JJWH.S106107.

20. Ginaldi L, De Martinis M. Osteoimmunology and beyond. Curr Med Chem 2016; 23(33):3754-74. doi: 10.2174/ 0929867323666160907162546.

21. De Laet $\mathrm{C}$, Kanis J, Odén A, Johanson H, Johnell O, Delmas P, et al. Body mass index as a predictor of fracture risk: a meta-analysis. Osteoporos Int 2005; 16(11):1330-8. doi: 10.1007/s00198-005-1863-y.
22. Bączyk G, Opala T, Kleka P, Chuchracki M. Multifactorial analysis of risk factors for reduced bone mineral density among postmenopausal women. Arch Med Sci 2012; 8(2):332-41. doi: 10.5114/aoms.2012.28562.

23. Bener A, Hammoudeh M, Zirie M, Heller RF. Is obesity a protective factor for osteoporosis? APLAR J Rheumatol 2005; 8(1):32-8. doi.org/10.1111/j.1479-8077.2005.00119.x

24. Amiri S, Behnezhad S, Hasani J. Body mass index and risk of frailty in older adults: A systematic review and meta-analysis. Obesity Medicine 2020:100196. 